hep-ph/9808462

CERN-TH/98-285

DFTT 55/98

\title{
ARE PARTON DISTRIBUTIONS POSITIVE?
}

\author{
Stefano Forte \\ INFN, Sezione di Torino, via P. Giuria 1, I-10125 Torino, Italy \\ Guido Altarelli \\ Theory Division, CERN CH-1211 Geneva 23, Switzerland \\ Dipartimento di Fisica "E. Amaldi", Università di Roma Tre, I-00146 Rome, Italy \\ and \\ Giovanni Ridolfi \\ INFN, Sezione di Genova, via Dodecaneso 33, I-16146 Genova, Italy
}

\begin{abstract}
We show that the naive positivity conditions on polarized parton distributions which follow from their probabilistic interpretation in the naive parton model are reproduced in perturbative QCD at the leading log level if the quark and gluon distribution are defined in terms of physical processes. We show how these conditions are modified at the next-to-leading level, and discuss their phenomenological implications, in particular in view of the determination of the polarized gluon distribution.
\end{abstract}

\author{
Invited talk given at \\ QCD98 \\ Montpellier, July 1996 \\ to be published in the proceedings
}

CERN-TH/98-285

August 1998

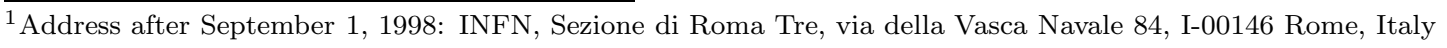




\title{
Are Parton Distributions Positive?*
}

\author{
Stefano Forte ${ }^{\mathrm{a} \dagger}$, Guido Altarelli ${ }^{\mathrm{b}}$, Giovanni Ridolfic \\ ${ }^{a}$ INFN, Sezione di Torino, via P. Giuria 1, I-10125 Torino, Italy \\ bTheory Division, CERN, CH-1211 Geneva 23, Switzerland \\ Dipartimento di Fisica "E. Amaldi”, Università di Roma Tre, I-00146 Rome, Italy

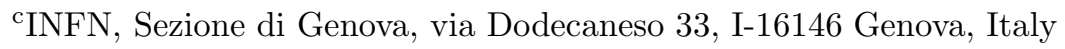

We show that the naive positivity conditions on polarized parton distributions which follow from their probabilistic interpretation in the naive parton model are reproduced in perturbative QCD at the leading log level if the quark and gluon distribution are defined in terms of physical processes. We show how these conditions are modified at the next-to-leading level, and discuss their phenomenological implications, in particular in view of the determination of the polarized gluon distribution.

In the naive parton model, parton distributions are viewed as probabilities for a parton with given momentum fraction to be found in its parent hadron, and are thus positive semi-definite. When extended to the polarized case, this simple picture leads to nontrivial constraints: since the polarized and unpolarized distributions $\Delta f$ and $f$ are sums and differences of helicity distributions,

$f=f^{\uparrow}+f^{\downarrow}, \quad \Delta=f^{\uparrow}-f^{\downarrow}$,

it follows that

$\left|\Delta f\left(x, Q^{2}\right)\right| \leq f\left(x, Q^{2}\right)$.

A minute's reflection shows that these relations cannot be true to all orders in perturbative QCD in a generic factorization scheme, since by choosing a scheme we are free to change $f$ and $\Delta f$ independently of each other in an arbitrary way, and thus the bound eq.(2) can be violated at will. This raises the question whether in perturbative QCD the bounds eq.(2) are valid at all, and more in general whether the helicity distributions of eq.(11) are necessarily positive semi-definite.

Let us consider first the simple case of the quark and antiquark distributions. These can be easily defined in terms of a physical process: pos-

\footnotetext{
*Presented by SF

${ }^{\dagger}$ Address after September 1, 1998: INFN, Sezione di Roma Tre, via della Vasca Navale 84, I-00146 Rome, Italy
}

itivity of the parton distribution can then be expressed in terms of the positivity of a physical cross section. Specifically, we can express the unpolarized and polarized deep-inelastic structure functions $F_{1}\left(x, Q^{2}\right)$ and $g_{1}\left(x, Q^{2}\right)$ in terms of quark, antiquark and gluon distributions as

$$
\begin{aligned}
F_{1}\left(x, Q^{2}\right) & =\frac{1}{2} \sum_{i=1}^{n_{f}} e_{i}^{2} C_{i}^{d} \otimes\left(q_{i}+\bar{q}_{i}\right) \\
& +2 n_{f}\left\langle e^{2}\right\rangle C_{g}^{d} \otimes g \\
g_{1}\left(x, Q^{2}\right) & =\frac{1}{2} \sum_{i=1}^{n_{f}} e_{i}^{2} \Delta C_{i}^{d} \otimes\left(\Delta q_{i}+\Delta \bar{q}_{i}\right) \\
& +2 n_{f}\left\langle e^{2}\right\rangle \Delta C_{g}^{d} \otimes \Delta g,
\end{aligned}
$$

where $C_{i}$ and $\Delta C_{i}$ are perturbatively computable coefficient functions.

Since the structure functions are in turn related to the asymmetry $A_{1}$ for deep-inelastic scattering of transversely polarized virtual photons on a longitudinally polarized nucleon through

$A_{1} \equiv \frac{\sigma_{1 / 2}-\sigma_{3 / 2}}{\sigma_{1 / 2}+\sigma_{3 / 2}}=\frac{g_{1}\left(x, Q^{2}\right)}{F_{1}\left(x, Q^{2}\right)}$,

it follows that $g_{1}$ is bounded by $F_{1}$ :

$\left|g_{1}\left(x, Q^{2}\right)\right| \leq F_{1}\left(x, Q^{2}\right)$

But at the leading log (LL) level all the quark coefficient functions in eq.(4) are equal to unity 
while all the gluon coefficient functions vanish:

$$
\begin{aligned}
& C\left(x, \alpha_{s}\right)=\sum_{k=0}^{\infty}\left(\frac{\alpha_{s}}{2 \pi}\right)^{k} C^{(k)}(x) ; \\
& C_{i}^{d,(0)}(x)=\Delta C_{i}^{d,(0)}(x)=\delta(1-x), \\
& C_{g}^{d,(0)}(x)=\Delta C_{g}^{d,(0)}(x)=0 .
\end{aligned}
$$

It follows that the bound eq.(5) immediately translates into the positivity condition

$$
\left|\Delta q_{i}\left(x, Q^{2}\right)\right| \leq q_{i}\left(x, Q^{2}\right),
$$

where the bound on each quark flavor separately is obtained by imposing that eq.(5) be true for any choice of target, i.e. for any value of the quark charges $e_{i}^{2}$ in eq.(四).

Beyond the LL level, the partonic positivity condition eq.(9) will only be satisfied in a parton scheme [1], where all coefficient functions retain their leading-order (LO) form eqs.(78). Such a scheme choice is however not necessarily advisable, since it preserves some arbitrarily selected partonic properties at the expense of other properties. In a generic scheme, instead, the partonic positivity condition will only hold at the LL level. Beyond LO, eq.(9) will in general be violated, and it will be replaced by a generalized condition, obtained substituting the full expression of $g_{1}$ and $F_{2}$ eq.(何) in the bound which relates them eq.(5). Accordingly, in a generic scheme the helicity parton distributions $f^{\uparrow \downarrow}$ eq. (1) are in general not positive semi-definite beyond LO.

Notice that this violation of positivity of parton distributions beyond $\mathrm{LO}$ is peculiar of the polarized sector. Indeed, in the unpolarized case the contributions $C^{(i)}$ to the coefficient functions beyond the first order need not be positive semidefinite - for example, all Mellin moments

$C\left(N, Q^{2}\right) \equiv \int_{0}^{1} d x x^{N-1} C\left(x, Q^{2}\right)$

of $C_{q}^{d,(1)}(x)$ with $N>1$ are negative. However, this is merely a subleading correction to the LO unpolarized coefficient function, thus within the region of validity of perturbation theory the full coefficient function $C\left(x, Q^{2}\right)$ remains positive semi-definite, and so does the unpolarized

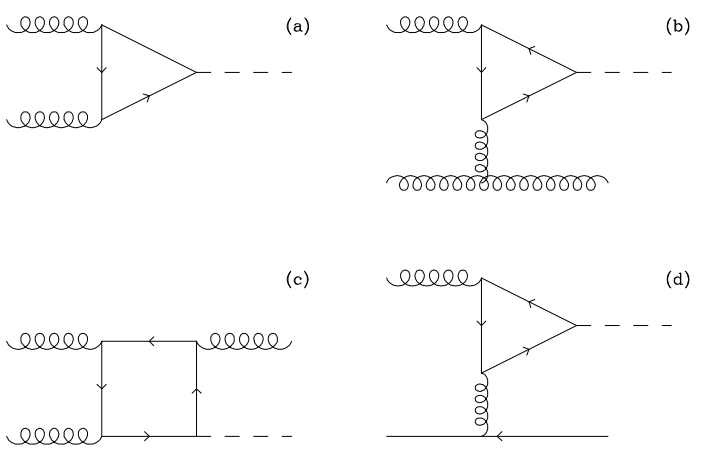

Figure 1. Diagrams for the processes (a) $g+g \rightarrow H$, (b) $g+q \rightarrow H+q,(\mathrm{c}, \mathrm{d}) g+g \rightarrow H+g$.

quark distribution as a consequence of the positiveness of the structure function $F_{1}$. On the other hand, whenever the bound eq.(5) is violated, either $f^{\uparrow}<0$ or $f^{\downarrow}<0$ : this may for instance happen if $\Delta C_{i}\left(x, Q^{2}\right)>C_{i}\left(x, Q^{2}\right)$ while the gluon contributions in eq.(11) are negligible.

In order to extend the above discussion to the case of the gluon distribution, we need a pair of polarized and unpolarized processes which at LO define the gluon, i.e. such that their LO coefficient functions are given by

$$
\begin{aligned}
& C_{i}^{h,(0)}(x)=\Delta C_{i}^{h,(0)}(x)=0 \\
& C_{g}^{h,(0)}(x)=\Delta C_{g}^{h,(0)}(x)=\delta(1-x) .
\end{aligned}
$$

An adequate choice [2] is the inclusive production of a scalar particle, such as the Higgs, in gluonproton collisions (fig. 1). The unpolarized and polarized cross-sections for this process can be written as convolutions of partonic cross sections with parton distributions in the proton:

$$
\begin{aligned}
\sigma[g p \rightarrow & H+X]\left(x, m_{h}^{2}\right)=A\left[C_{g}^{h} \otimes g\right. \\
& \left.+C_{s}^{h} \otimes \Sigma\right] \\
\Delta \sigma[g p \rightarrow & H+X]\left(x, m_{h}^{2}\right)=A\left[\Delta C_{g}^{h} \otimes \Delta g\right. \\
& \left.+\Delta C_{s}^{h} \otimes \Delta \Sigma\right]
\end{aligned}
$$

where $A$ is a dimensionful coefficient, $x \equiv \frac{m_{h}^{2}}{s}$ and the singlet quark distribution is defined as $\Sigma\left(x, Q^{2}\right)=\sum_{i=1}^{n_{f}}\left[q_{i}\left(x, Q^{2}\right)+\bar{q}_{i}\left(x, Q^{2}\right)\right]$ (and likewise for the polarized distribution $\Delta \Sigma$ ). Now at LO (fig. 1a) momentum conservation implies that the partonic cross section is $\sigma(g g \rightarrow H)=$ $C \delta\left(x s-M_{H}^{2}\right)$, while helicity conservation implies 
that the helicity of the incoming gluon and the gluons in the proton must be the same (in a collinear frame). This means that the LO coefficient functions have the form eq. (11, 12 , and then the requirement

$$
|\Delta \sigma[g p \rightarrow H+X]| \leq \sigma[g p \rightarrow H+X]
$$

implies that the polarized and unpolarized gluon distributions satisfy the bound eq.(2). Beyond LO more partons can be emitted in the final state (fig. 1b-d), the coefficient functions are generic, and the LO bound is again replaced by a generalized condition, found using the explicit expression of the cross section eq.(14) in the cross-section positivity bound eq.(14).

At next-to-leading order (NLO) the pair of naive bounds of the form eq.(2) satisfied by the quark singlet and gluon distribution are thus replaced by a pair of bounds which mix these two distributions through the coefficient functions of the processes that define them at LO. The bounds simplify by taking moments [as in eq.(10)], which turn the convolutions in eqs.(河14) into ordinary products: for instance, the NLO bound on the gluon becomes

$$
\frac{\left|\Delta g\left(1+\frac{\alpha_{s}}{2 \pi} \Delta C_{g}^{h,(1)}\right)+\frac{\alpha_{s}}{2 \pi} \Delta C_{s}^{h,(1)} \Delta \Sigma\right|}{g\left(1+\frac{\alpha_{s}}{2 \pi} C_{g}^{h,(1)}\right)+\frac{\alpha_{s}}{2 \pi} C_{s}^{h,(1)} \Sigma} \leq 1,
$$

where the various parton distributions and coefficient functions depend on the Mellin variable $N$ and the scale $Q^{2}$.

Given the unpolarized parton distributions, which are in general much better known than the polarized ones, the bounds thus take the form of allowed areas in the $\Delta \Sigma(N), \Delta g(N)$ plane. These can be determined to NLO with the above choice of defining processes, since the DIS coefficient functions are well known [3], while the unpolarized [4] and polarized [2] coefficient functions for the process of fig. 1 have been determined recently. The bounds (using CTEQ4LQ [5] unpolarized distributions) are shown in fig. 2 for selected values of $N$. The bounds are only significant for $N>1$, because at $N=1$ the unpolarized parton distributions in the denominator of eq.(15) diverge. Because the unpolarized coefficient functions in the denominator of eq. (15)
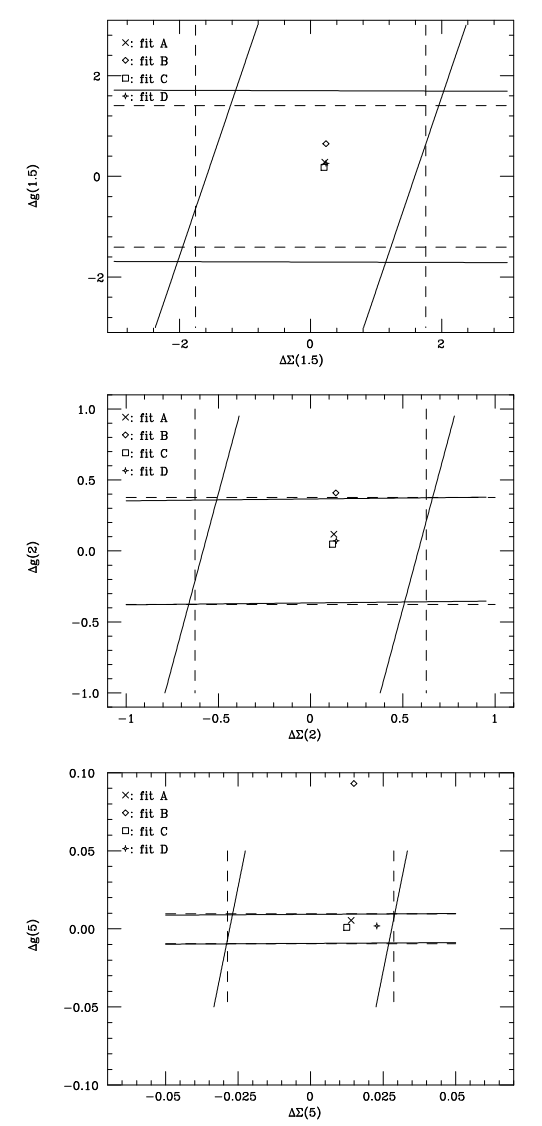

Figure 2. The LO (dashed lines) and NLO (solid lines) positivity bounds on $\Delta \Sigma(N)$ and $\Delta g(N)$ for $Q^{2}=1 \mathrm{GeV}^{2}$ and $N=1.5,2,5$. The values of $\Delta \Sigma$ and $\Delta g$ corresponding to the NLO fits to $g_{1}$ data of ref. [6] are also shown.

also diverge as $N \rightarrow 1$, the NLO bound on $\Delta g$ is much less restrictive than the naive LO one if $N$ is close to one. However, when $N$ grows the NLO bound becomes somewhat more restrictive than the LO one, and eventually for very large $N$ the difference between LO and NLO is negligible.

We may further use the bounds on the moments of the polarized gluon distribution to construct a maximal gluon distribution, i.e. $\Delta g_{\max }\left(x, Q^{2}\right)$ such that $\left|\Delta g\left(x, Q^{2}\right)\right| \leq \Delta g_{\max }\left(x, Q^{2}\right)$, by fixing the polarized quark distribution and performing numerically the Mellin inversion of the bound eq.(15) (fig 5).

Comparison of the NLO bounds on $\Delta g$ thus obtained from the positivity constraint with the results of a recent NLO fit to $g_{1}$ data [6] (fig. 3) un- 

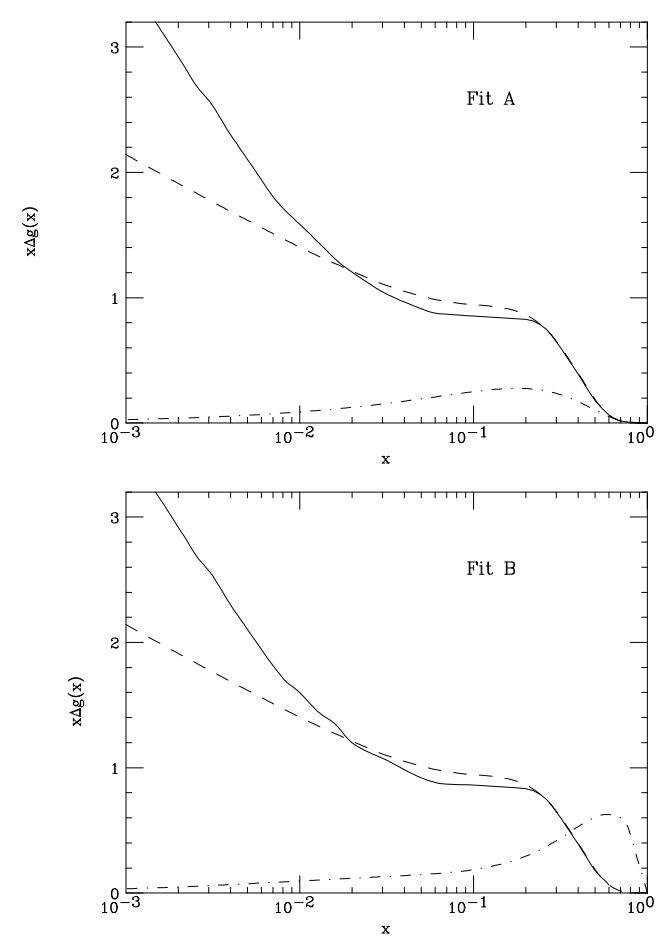

Figure 3. The maximal gluon distribution at $Q^{2}=$ $1 \mathrm{GeV}^{2}$ obtained from the LO (dashed lines) and NLO (solid lines) positivity bounds using the polarized quark distributions corresponding to fits $\mathrm{A}$ and $\mathrm{B}$ of ref. [6]. The corresponding best-fit polarized gluon distribution from ref. [6] is also shown (dot-dashed).

derscores their phenomenological relevance. Indeed, the polarized gluon distribution can be currently determined [6] only from scaling violations of $g_{1}$,

$$
\begin{aligned}
& \frac{d}{d t} g_{1}^{\text {singlet }}\left(N, Q^{2}\right)= \\
& \quad=\frac{\left\langle e^{2}\right\rangle}{2} \frac{\alpha_{s}}{2 \pi}\left[\gamma_{q q} \Delta \Sigma+2 n_{f} \gamma_{q g} \Delta g\right]+O\left(\alpha_{s}^{2}\right)
\end{aligned}
$$

These, however, only allow a determination of small moments of $\Delta g$, while high moments are essentially unconstrained, due to the fact that at large $N\left|\gamma_{q g}\right| \ll\left|\gamma_{q q}\right|$ (see fig. 4). As a consequence, the data can be described equally well by polarized gluon distributions whose higher moments are widely different, provided their low moments remain more or less the same. This is the case of the $A$ and $B$ fits of ref. [6] displayed in fig. 3. The corresponding uncertainty is thus especially noticeable in the large $x$ region. Whereas

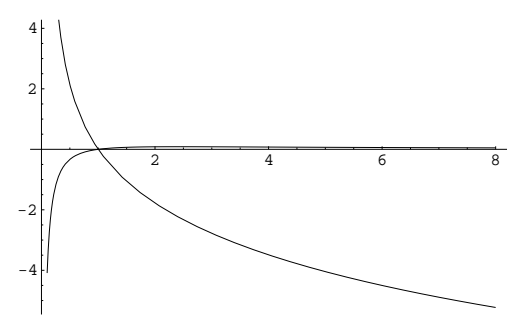

Figure 4. The LO anomalous dimensions $\gamma_{q q}(N)$ and $\gamma_{q g}(N)$ (top to bottom at small $N$ ) as a function of $N$.

this is not important if one is only interested in a determination of first moments, it prevents a good determination of the shape of $\Delta g$. The positivity bounds, by providing an independent constraint on higher moments allow to substantially improve the determination of the shape of $\Delta g$.

To this purpose it is important to understand at which scale the bounds should be imposed. Indeed, it is easy to see that

$$
\lim _{Q^{2} \rightarrow \infty} \frac{\left|\Delta q_{i}\left(N, Q^{2}\right)\right|}{q_{i}\left(N, Q^{2}\right)}=\frac{\left|\Delta g\left(N, Q^{2}\right)\right|}{g\left(N, Q^{2}\right)}=0 .
$$

It follows that the LO bounds eq.(2) will always be satisfied at large enough scale, but conversely they will always be violated at a low enough scale. Correspondingly, at low scale the NLO deviation from the LO bounds will blow up. Hence, positivity bounds at large scales are trivial, but they should not be imposed at very low scale either, where they are unreliable. However, if imposed at the boundary of validity of perturbation theory, positivity bounds can be phenomenologically relevant in providing complementary information which is useful in the determination of the shape of polarized parton distribution.

\section{REFERENCES}

1. G. Altarelli, R. K. Ellis and G. Martinelli, Nucl. Phys. B143 (1978) 521; B157 (1979) 461.

2. G. Altarelli, S. Forte and G. Ridolfi, hep-ph/9806345

3. W. A. Bardeen et al, Phys. Rev. D18 (1978) 3998. J. Kodaira, Nucl. Phys. B165 (1980) 129.

4. R. K. Ellis et al., Nucl. Phys. B297 (1988) 221. S. Dawson, Nucl. Phys B359 (1991) 283.

A. Djouadi, M. Spira and P. M. Zerwas, Phys. Lett. B264 (1991) 440.

5. H. L. Lai et al., Phys. Rev. D55 (1997) 1280.

6. G. Altarelli, R. D. Ball, S. Forte and G. Ridolfi, Nucl. Phys. B496 (1997) 337; Acta Phys. Pol. B29 (1998) 1145, hep-ph/9803237. 